\title{
MultiNet: An Intelligent Sensor Cable
}

\author{
Stephan Sommer*, Alois Knoll*, Christian Buckl ${ }^{\dagger}$, Gerd Bauer ${ }^{\ddagger}$ and Jens Schulz ${ }^{\S}$ \\ *Technische Universität München \\ Boltzmannstr. 3, D-85748 Garching, Germany \\ \{sommerst,knoll $\} @$ in.tum.de \\ ${ }^{\dagger}$ fortiss $\mathrm{GmbH}$ \\ Guerickestr. 25, D-80805 München, Germany \\ buckl@fortiss.org \\ $\ddagger$ efm-systems GmbH \\ Reinsburgstrasse 96/1, D-70197 Stuttgart, Germany \\ bauer@efm-systems.de \\ $\S$ angaris $\mathrm{GmbH}$ \\ Grosse Maerkerstr. 18, D-06108 Halle / Saale, Germany \\ jens.schultz@angaris.de
}

\begin{abstract}
In all areas of industrial production and construction, companies have to implement processes with increasing complexity to satisfy their customer's needs in respect of product quality and continuity. To fulfil these requirements, a continuous process monitoring and documentation is required to assure the process quality but without increasing production cost significantly. In our project we develop an intelligent sensor cable which can be easily integrated into industrial processes using industrial communication standards like ProfiBus. To support an easy installation process of our sensor cable, it needs to be self contained; in our case the sensors and the processing unit, required for pre-processing and sensor management, are integrated in the cable. To increase usability, the cable can also be tailored by the customer to the required length. The key application of our sensor cable is the documentation of the concrete curing processes using the integrated temperature sensors for safety critical constructions.
\end{abstract}

\section{INTRODUCTION}

In common control and automation scenarios in industry, sensors are usually directly connected to $\mathrm{PLCs}^{1}$ to gather the data required for process control. In many of the scenarios where process documentation is required, the documentation is derived from the data gathered by the PLCs simply by connecting them to a backend where their sensor readings and additional information calculated by the PLCs are stored in some kind of database; this database provides an easy to use interface for management and for people responsible for the product quality.

This approach has a major drawback: The sensing for process documentation is not implemented separately of the sensing required for process control. If the sensors of the PLC read wrong values, this affects the process control and by that the commands issued by the PLC. In addition, the documentation written to the database only shows that the PLC processed the data in the right way. Without additional redundancy the result can be a broken process where gathered data suggests a correct one. To get an independent instance

\footnotetext{
${ }^{1}$ Programmable logic controller
}

for the production documentation, an individual sensor network only responsible for the documentation is required. This approach allows deploying sensors individually from the sensors required for the process control. Because these sensors gather only data which is necessary to monitor the quality of the process, they can be placed to optimal locations without influencing the process control. This also allows the adaption and extension of the sensor network without touching the PLCs responsible for the process control. This fact is especially important for service providers which only provide solutions for the documentation and do not want to get in touch with the process control for warranty reasons. Of course the data gathered by the PLC can also be integrated in the database to enhance the informative value of the complete process data. The data gathered by both systems can also be compared to allow an estimation of the sensor data quality and can of course be used to detect problems in the process. Furthermore, an easy integration of the sensor network to the remaining infrastructure needs to be guaranteed. The integration can be realized by usual low level industrial interfaces like ProfiBus[1] or DeviceNet[2] but also using higher level interfaces known from the business world (e.g. Web services)[3].

Due to the fact that in industrial production the cost factor is essential, the sensor networks need to be easy deployable, easy to integrate and self contained (the control and management-logic is inside the sensor-network and an industry standard connector and protocol is provided for simple integration in deployed applications).

The systems can of course also be deployed as pure sensor network without helping to control processes but only for monitoring the environment of stored products and chemical processes. The availability of these intelligent sensors and the reasonable low price also allows the installation of such sensor-networks in scenarios, where beforehand no sensors have been used or where sensor deployment was error-prone and complicated. In our paper, we present an intelligent 


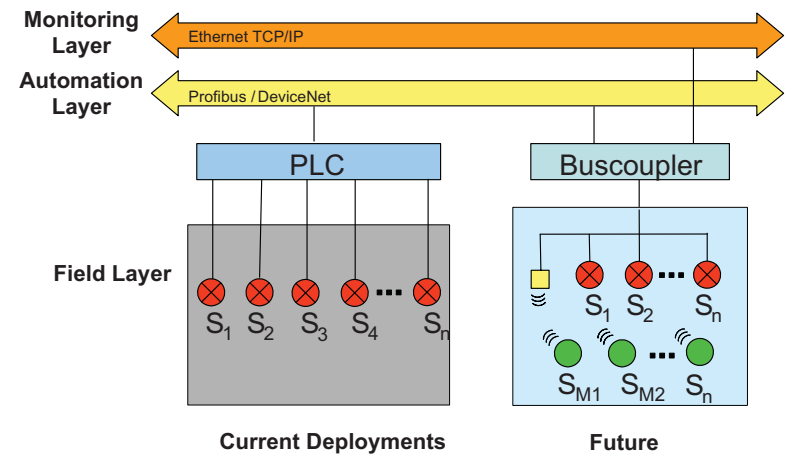

Fig. 1. Automation System Architecture

sensor cable for temperature measuring which is easy and cheap to integrate in the manufacturing process like concrete construction.

The remainder of this paper is structured as follows: A common system architecture for sensor networks in control applications is described in section II. Two fields of application for our sensor cable are discussed in section III and the design process of our sensor cable is elaborated in section IV. Finally the paper is concluded with a summary of the results and plans for further work in section $\mathrm{V}$.

\section{System ARChitecture}

A classical design pattern for control applications in industry is to directly connect sensors to a PLC. This prevents system designers from using these sensors in more than one application. As already discussed in the introduction, flexible systems are required for future deployments. An overview of requirements and state of the art in industrial sensor networks (wired and wireless) is given in [4]. Already deployed sensors need to be easily integrated in new applications as well as newly deployed sensors need to be integrated in already running applications. Both scenarios are depicted in Fig. 1. In the automation domain, deployments can be divided into three layers. The lowest layer is the FieldLayer where local control tasks are performed by PLCs and where the sensors are located. The Second layer is the AutomationLayer. In this layer, the communication between PLCs is realized and longer distances can be covered. The MonitoringLayer provides the interfaces to business applications like process planning and management tools. The bottom left of Fig. 1 shows a common automation scenario with sensors directly connected to a PLC, whereas the sensors on the right are connected to an industrial bus using bus couplers. The right design increases flexibility by permitting the users to integrate sensors in multiple applications. The next level of abstraction for developing sensor network applications can be a uniform sensor network gateway providing the sensor data to customers of all kind located at the MonitoringLayer e.g. control applications as well as business applications. Using this approach, the sensors become a service provided by the sensor network for all kinds of subscribers. The feasibility of a service oriented approach (SOA) for sensor networks is discussed in [5]. When extending the flexibility of sensor usage, this always comes with an increased system complexity. Especially when introducing mobile wireless devices, a redesigned stack is required. One feasible approach is a high level programming abstraction provided by PhysicalNet[6]. In addition of allowing multiple applications to use one sensor, they also provide concepts for moving devices by introducing Gateways and Negotiators to raise the level of abstraction. Summing up, to provide easy and flexible access to measurements, a well planned infrastructure is required.

\section{FIELDS OF APPLICATION}

In the early phase of our project, we identified two different applications for our sensor cable equipped with temperature sensors. These two application scenarios are characterized by the harsh environment where the measuring is done and will be elaborated in this section.

\section{A. Concrete Temperature Monitoring}

When building constructions with concrete like tunnels (see Fig. 2) or bridges, it is crucial that the complete building process works as desired to insure the stability of the construction for many years. For the awarding authority it is necessary to check the quality of construction and for the construction companies, it is necessary to prove that they did well in case of defects. So both parties in the construction process can benefit from a doubtless documentation.

For the process of concrete building, one key indicator of the process quality is the temperature gradient while concrete hardening[7]. When recording this profile, this can show, that the guidelines are met. The recording of temperatures is not a new kind of documentation invented by us, but by using our sensor cable, it can be performed much easier. In the common way of measuring - without our sensor cable - the construction workers needed to place individual sensors every three meter into the concrete. Each of these sensors has three wires (two for power, one for the temperature represented by a voltage between $0 \mathrm{~V}$ and $5 \mathrm{~V}$ ) which needed to be individually connected to a data logger. For one segment of approximately 50 meter, 16 sensors are necessary and therefore 48 individual 


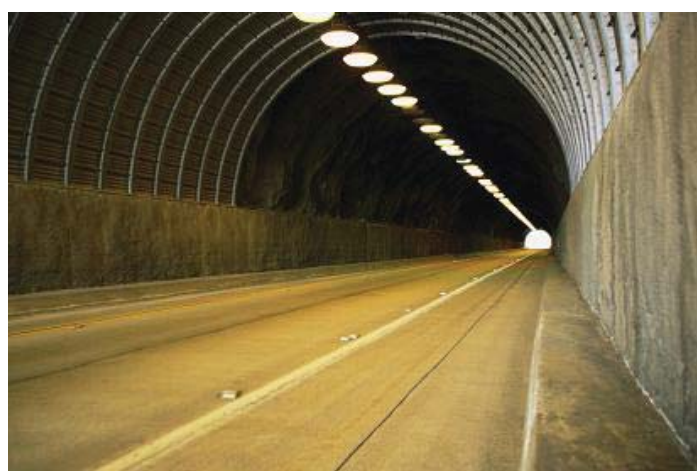

Fig. 2. Application: Tunnel Construction

wires need to be correctly connected to a data logger. This is a very error prone and time consuming task. Experience shows, that most of the time only two thirds of the sensors are correctly connected and can be used for data logging. In contrast to this approach, our cable contains the 16 sensors and electronics for data pre-processing. So instead of the 48 individual wires for the common approach, we only need to connect one easy to use but robust industrial connector which houses the four wires; two wires for power supply and two wires for the communication with the 16 sensors respectively for the communication with multiple concatenated cables to increase the data points in one segment or to measure in multiple segments simultaneously.

\section{B. Temperature Monitoring in Drill Holes}

When monitoring the temperatures in drill holes, we are facing similar problems as in the concrete building scenario. We need to record the temperature in different layers of the soil to document the effects of global warming or to monitor activity in earth's interior. In the common way, a steel cable was manually equipped with sensors (all of them with at least three wires per sensor) every three meter and the wires of the sensors were taped to the steel cable. Finally all these individual wires have been connected to a data logger. This solution was hard to handle especially when the cable was put into the hole to avoid sensor and cable damage. In addition, the diameter of steel cable is increased by the cables of the sensors attached to the side of the steels cable.

Using our sensor cable, depending on the tensile strength of the outer layer of our cable, the steel cable can even be avoided, or at least, there is only one small cable which needs to be attached to the steel one. This reduces possible errors because the cabling process is now much simpler. As in the concrete building scenario, there is again only one robust connector at the end of the cable where a data logger and the power supply can be connected. Of course, if more than one segment is needed to monitor to greater depth, several cables can be connected up to a theoretical length of approximately 500 meter.

\section{The Intelligent CABle}

This project is not only research driven so the requirements needed to be collected and the design decisions were made to come up with a market ready product within two years. In this section, we show the steps we performed until now to develop the sensor cable. After collecting the design requirements, two concept prototypes were developed and evaluated. Based on these results, a system prototype was built.

\section{A. Design Requirements}

When starting a development project, bringing together potential users and manufacturers is the fist step. During the requirements engineering phase, we tried to come up with a specification suitable for all project partners and application scenarios. One key requirement given by the customers and future users was that the deployment of our sensor cable must be simple. It is a key requirement, that a construction worker can install the cable without the help of a special technician. To guarantee a simple operation and installation, the additional external components need to be reduced to the minimum. In ideal case, there is only the sensor cable and a data-logger which is battery powered and also capable of supplying the sensor cable. To keep the data logger and with it the batteries as small as possible, the energy consumption of the sensor cable needs to be as small as possible to allow a high lifetime at a low weight and size.

To allow a simple check of operability, the data logger needs to provide some indication for the user like some LEDs or a display. Especially for the process-automation community, an important aspect is the simple integration into already deployed applications. For most of the customers, the durability of the sensor cable and the additional equipment in the harsh environments of a construction site is also a key factor. The sensitive electronics needs to be protected from water, mud and dust, especially in the concrete temperature monitoring scenario where concrete is thrown at the wall where the cable is mounted. To cope with this harsh environment, many precautions need to be taken into account e.g. for the connectors, the cable hull and the housing of the data logger. For efficient use of our sensor cable, it was also a key requirement to support at least a length of 50 meters. This is long enough to monitor one construction segment, but also short enough for efficient handling. To provide a solution to monitor longer segments, the cable needs to be concatenable to increase the cable length. Depending on the customer's needs, there need to be up to 10 segments concatenatenable. For special needs, the cable segments can also be shortened to the length specified by the customer. To improve the cable handling and to reduce the risk of damaging the cable, it has to be small and flexible enough to be stored on a bowl but also thick enough to provide enough protection for the integrated electronics.

Last but not least, the total cost of the cable (for the purchase, the installation and maintenance) is also a reasonable requirement to convince customers to use the sensor cable. Although considering our primary application, the sensor cable will be 
put into concrete and so be only used once, the data logger needs to be re-usable.

\section{B. Cable Layout}

Considering the different requirements given by our partners and the future customers, we elaborated two different designs for our sensor cable. The first approach depicted in Fig. 3 is to place several micro controller units (MCUs) inside the cable and connect up to four sensors to one MCU. The second approach depicted in Fig. 5 is to house the MCU inside the cable connector and to use bus-capable sensors. In the following we will elaborate these two different solutions and conclude this section with our current implementation.

1) Design 1: MCUs Inside the Sensor Cable: Due to the lack of bus capable sensors which could be explicitly addressed inside the cable when we started the project, our first approach was to use TSIC306[8] digital sensors and connect four of them to each MCU in the sensor cable. We selected four sensors per MCU as a trade-of between wiring complexity (each sensor needs power supply and communicaton lines) MCU cost and achievable sampling rate. The MCUs are connected using a single master RS485 bus[9]. This implementation distributing the sensing and pre-processing load across many MCUs and uses sensors we already had experience with. A further aspect why the use of multiple MCUs has advantages, is, that the measured data can be cached (e.g. for pre-processing) over a longer period of time, because one MCU is only responsible for up to four sensors. In Fig. 4 you can see the first prototype of our setting already equipped with four sensors.

Although this implementation could fit the requirements,

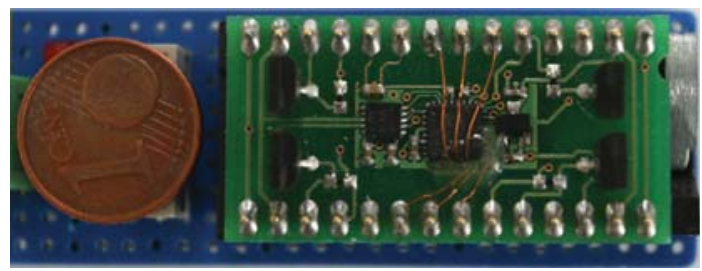

Fig. 4. First Prototype with TSIC-Sensors for Protocol Tests

it also comes with several drawbacks. Because of the high number of MCUs involved, the sensor cable becomes a highly distributed system of nodes. The challenges start immediately after powering up the sensor cable. The number of MCUs inside the cable need to be detected and their unique identifier need to be read, preferably not by polling the complete ID number range. A further aspect making the development complex was the limited selection of MCUs small enough to be placed inside the cable and powerful enough be programmed in $\mathrm{C}$ and to parse the digital sensor protocol; in our case the ZACwire[10] protocol. A highly distributed system also increases the complexity of software development, especially debugging. In the development process, the software developer also needs to make sure, that in each code refinement step all MCUs inside the cable get updated. Therefore an infrastructure is required to do this in a fail-save and efficient way. In addition, for the pre-processing, the MCUs need to be capable to store a local history of measurements. Having in mind, that each sensor needs to be connected individually to the MCU, the complexity of the manufacturing process also increases dramatically. When taking into account, that the sensor cable needs to be powered from battery for several days, the number of MCUs can also cause problems although they provide energy saving capabilities.

To summarize, this was our first approach which forms a usable implementation but comes with several drawbacks. To cope with these drawbacks, we again started to scan the market for sensors and finally found a sensor which helped us to dramatically reduce system complexity.

2) Design 2: $M C U$ inside the Connector: With the new type of sensor providing a bus interface, we are now able to significantly cut down complexity in manufacturing and software development. In contrast to the implementation with the MCUs located directly inside the cable, we are now able to reduce the number of MCUs to one for each cable segment and additionally move this MCU into the connector. For a schematic overview of the layout, see Fig. 5. This design change allows us to select a MCU based on the requirements with respect to processing power and data storage capabilities sufficient for data-history. The size constraints can now almost be ignored. This new design also allows to easily provide different versions of our system using different MCUs without changing the cable manufacturing process. It is now possible to use a MCU with integrated CAN interface or even with Ethernet if the connector is allowed to be big enough to house the MCU. All this freedom to customize the measuring system can be provided without making changes to the cable itself. The significant reduction of MCUs also supports us in reducing the energy consumption and makes development and testing easier by reducing the complexity of the software. For our tests, we started using the test circuit depicted in Fig. 7 equipped with three sensors.

Regarding all these advantages of the new design, there are

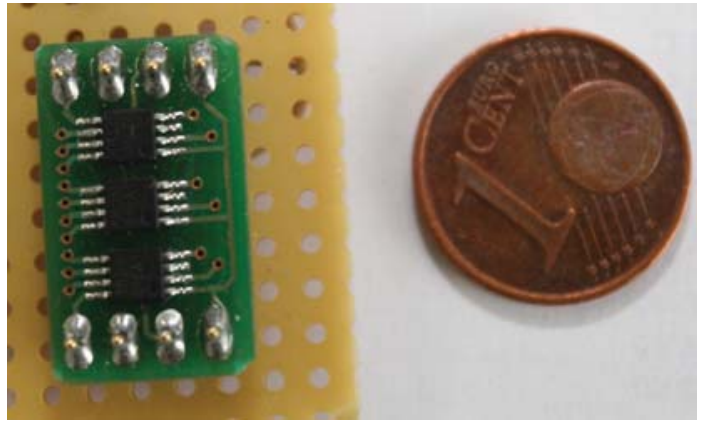

Fig. 7. First Prototype with Bus-Sensors for Protocol Tests

also some drawbacks. In contrast to the sensors integrated in the first design, we do not have experience in the durability and longevity of the selected sensors. Therefore we will have to do additional tests in our test bed and in the first installation. 


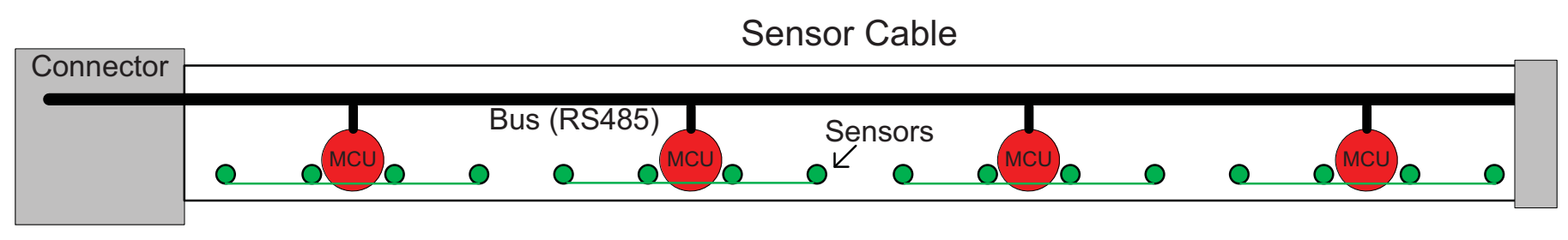

Fig. 3. Sensor Cable with integrated MCUs

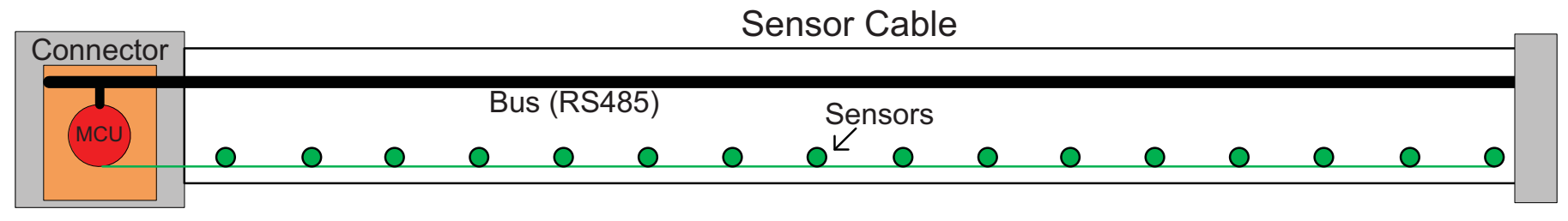

Fig. 5. Sensor Cable with MCU in Connector

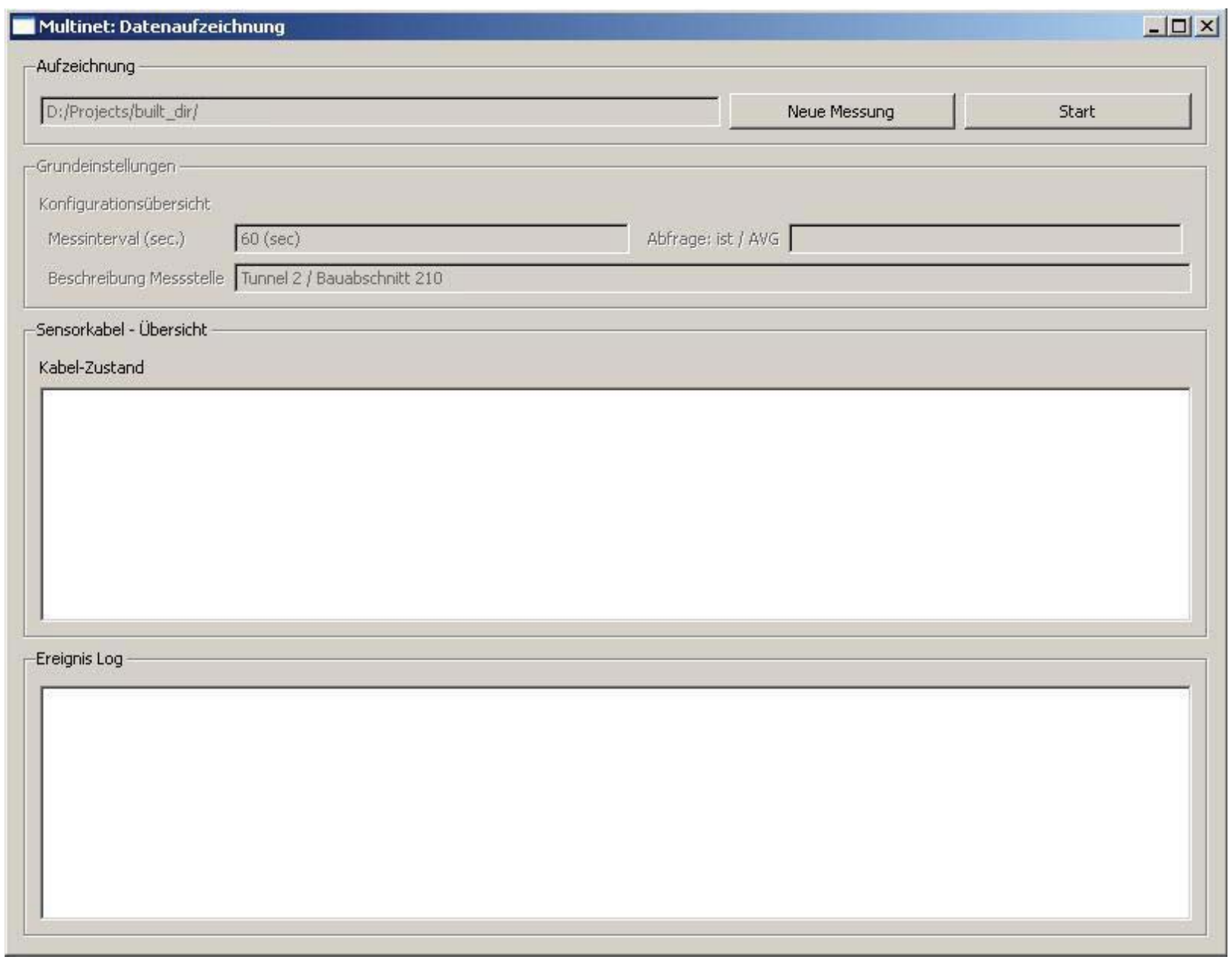

Fig. 6. Screenshot of PC based Data Logger 


\begin{tabular}{|l|c|c|}
\hline & Design 1 & Design 2 \\
\hline Manufacturing complexity & very high & average \\
MCU constraints & very high & almost none \\
Number of MCUs / Segement & 4 & 1 \\
Number of Sensors / MCU & 4 & 16 \\
Maximum Sensors / Segment & $\infty$ & 16 \\
Communication Protocol & RS485 & RS485, CAN, Ethernet, ... \\
In-cable Data pre-processing & none & avg, min, max... \\
SW development complexity & high & low \\
\hline
\end{tabular}

TABLE I

COMPARISON OF BOTH DESIGNS

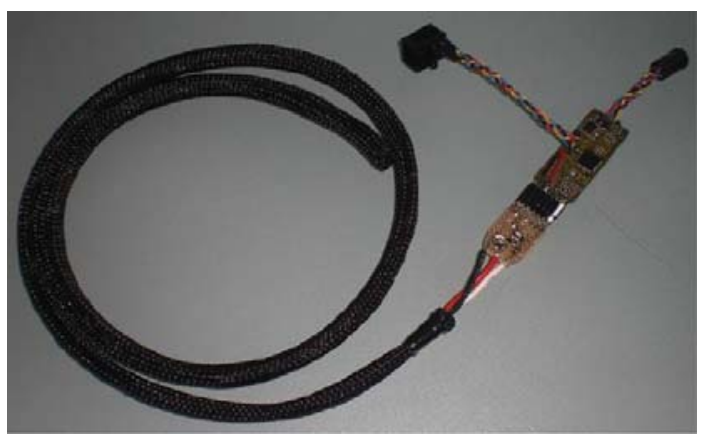

Fig. 9. Hand-Crafted Prototype of Cable in a Flexible Hose with MCU

b) Cable Layout: The prototype cable consists of seven wires. As already mentioned, two are required for the cable power supply $(12 \mathrm{~V} \ldots 24 \mathrm{~V}$ DC) and additional two wires are required for the MODBUS communication over the serial RS485 bus. In addition to these wires, there are additional wires to connect the sensors to the MCUs. Two of these additional wires are required for the sensor power supply and one is required for the communication between sensors and MCU. These wires are twisted and then packed into a flexible hose to form the first prototype (see Fig. 9). For the final product, the cable will be housed in e.g. PVC coat.

c) Supported Features: To increase the system reliability, it is important to detect problems as fast as possible. We decided to detect the connected sensors inside the cable after each system start. In addition, this action can also be performed from time to time. This procedure allows us to recognize, if sensors can not be reached anymore, even after a restart. To increase the system reliability, a CRC is used to secure the communication between sensors and MCU as well as between MCU and data logger.

In addition to these low level features, we also implemented features for data pre-processing and error-logging. The user can e.g. decide that the temperature provided to the data logger should be the average of up to 32 measurements or that the average of all measurands of the cable should be provided at a defined MODBUS register. To allow a flexible adaption to the user requirements, the sampling rate can also be configured by the user and stored permanently for further use. For error logging and tracking, the user can specify upper and lower boundaries for legal measurands. If these are violated, a log entry is made. These logs can also be inspected by the user.

d) Further Development Phases: After the first tests in our lab with a software data logger installed on a notebook (screenshot depicted in Fig. 6) and with an external power supply, we will continues to optimize the power consumption to allow a sufficient long period of battery powered operation. In addition, we also plan to provide a data logger capable of harsh environments to form a complete bundle for future customers.

\section{Concatenation of Cables}

To provide cables with a greater length than one segment can provide, the cables need to be concatenable. By using the 
RS485 bus to interconnect the MCUs, this requirement can be easily fulfilled by selecting different Modbus IDs for the controllers to avoid address conflicts. The cables can simply be concatenated using the installed connectors on both ends. The data processing and the error handling are done individually in each segment, the total bandwidth of the RS485 bus is divided by the number of segments connected.

\section{CONCLUSION}

In this paper, we presented an intelligent sensor cable tailored for temperature measurement. We discussed two possible implementations of such a cable, elaborated the reason why we selected our approach and finally presented our prototype. The key feature of our approach is the easy integration in already established processes, the simple handling - even in harsh environments - and the low price. In comparison to the common approach in our fields of application, a significant speedup in deployment can be achieved and errors in the installation process can be minimized. By using a data-logger and our sensor cable, the use of expensive PLCs can be avoided or reduced to a minimum. For the current version, we only support temperature measuring but there could also be additional applications fields using our approach of moving the intelligence into the cable. For further work, we plan to do in depth testing in the main area of application - the tunnel construction - to verify the results from our lab-tests in a real world setting. We are also working on a graphical deployment and management tool for the sensor network configuration and maintenance.

\section{REFERENCES}

[1] PROFIBUS Nutzerorganisation e. V. (PNO), Haid-und-Neu-Str. 776131 Karlsruhe Germany, "Profibus - easy to use process automation."

[2] ODVA, "Devicenet - cip on can technology," 2008.

[3] C. Buckl, S. Sommer, A. Scholz, A. Knoll, A. Kemper, J. Heuer, and A. Schmitt, "Services to the field: An approach for resource constrained sensor/actor networks," in The Fourth Workshop on Service Oriented Architectures in Converging Networked Environments (SOCNE), 2009.

[4] A. Flammini, P. Ferrari, D. Marioli, E. Sisinni, and A. Taroni, "Wired and wireless sensor networks for industrial applications," Microelectronics Journal, vol. 40, no. 9, pp. 1322-1336, 2009.

[5] A. Scholz, C. Buckl, S. Sommer, A. Kemper, A. K. an d Jörg Heuer, and A. Schmitt, "eSOA - service oriented architectures adapted for embedded networks," in Proceedings of the 7th International Conference on Industrial Informatics), Jun. 2009.

[6] P. Vicaire and J. Stankovic, "Physicalnet: Cross-network applications for multi-user sensor and actuator networks," ACM SIGBED Review, vol. 5, pp. 1-2, 2008.

[7] A. Schindler and B. Frank McCullough, "Importance of concrete temperature control during concrete pavement construction in hot weather conditions," Transportation Research Record: Journal of the Transportation Research Board, vol. 1813, no. -1, pp. 3-10, 2002.

[8] ZMD AG, "Tsic 306 - high precision, rapid response, low cost temperature sensor ic," Feature Sheet, 2005.

[9] B\&B Electronics Mfg. Co. Inc., "Rs-422 and rs-485 application note," 2006.

[10] IST AG, "Ist tsic temperature sensor ic - application notes zacwire digital output."

[11] Microchip Technology Inc., "Pic18f2585/2680/4585/4680 data sheet 28/40/44-pin enhanced flash microcontrollers with ecan technology, 10bit a/d and nanowatt technology," 2007.

[12] Modbus.org, "Modbus over serial line specification \& implementation guide v1.0," 2002. 